\title{
Role of RLIP76 in doxorubicin resistance in lung cancer (Review)
}

\author{
RIT VATSYAYAN, PANKAJ CHAUDHARY, POORNA CHANDRA RAO LELSANI, \\ PREETI SINGHAL, YOGESH C. AWASTHI, SANJAY AWASTHI and SHARAD S. SINGHAL \\ Department of Molecular Biology and Immunology, University of North Texas \\ Health Science Center, Fort Worth, TX 76107, USA
}

Received January 19, 2009; Accepted March 13, 2009

DOI: 10.3892/ijo_00000279

\begin{abstract}
Lung cancer is still a major cause of cancer deaths in spite of considerable efforts in its systemic therapy. Chemotherapy, along with local irradiation is frequently employed but as a palliative therapy. Inherent and acquired resistance in NSCLC and SCLC towards chemotherapeutic agents further makes chemotherapy an incommodious problem. The resistance mechanisms responsible for inherent DOX-resistance of NSCLC and acquired DOX-resistance in SCLC have been the subject of numerous investigations. This review will focus on the recent studies done for understanding the mechanism(s) of inherent and acquired resistance in NSCLC and SCLC and how these can be exploited for the future development of more effective novel biologic agents for the treatment of lung cancer.
\end{abstract}

\section{Contents}

1. Introduction

2. RLIP76: a non-ABC transporter

3. Physiological significance of RLIP76

4. Doxorubicin resistance in lung cancer

5. RLIP76 is a major DOX transporter

6. Impairment of transport of RLIP76 sensitizes lung cancer cells to doxorubicin

Correspondence to: Dr Sharad S. Singhal, Department of Molecular Biology and Immunology, 3500 Camp Bowie Blvd., University of North Texas Health Science Center, Fort Worth, TX 76107-2699, USA

E-mail: ssinghal@hsc.unt.edu

Abbreviations: RLIP76 (RalBP1), Ral-interacting protein; GSH, glutathione; GS-E, glutathione-electrophile conjugates; DNP-SG, dinitrophenyl S-glutathione; DOX, doxorubicin; 4HNE, 4-hydroxyt-nonenal; IOVs, in-side-out vesicles; MDR, multi-drug resistance; Pgp, P-glycoprotein; MRP1, multi-drug resistance associated protein; SCLC, small cell lung cancer; NSCLC, non- small cell lung cancer; POB1, partner of RLIP76

Key words: RLIP76, PKC $\alpha$, cancer, drug-resistance, glutathioneconjugate transport, doxorubicin
7. Role of PKC $\alpha$ and RLIP76 in transport-mediated DOXresistance in lung cancer

8. RLIP76 is essential for PKC $\alpha$ mediated DOX-resistance

9. RLIP76 inhibition by POB1 and Hsf-1 induces DOX sensitivity

10. Conclusions

\section{Introduction}

Lung cancer is the most frequent lethal neoplasm in the world and is the leading cause of cancer deaths in the United States. It represents $12 \%$ of all cancers and $18 \%$ of cancers deaths world wide $(1,2)$. Because of the lack of widely applicable screening methods, it is diagnosed usually in relatively advanced stages for which only palliative therapy can be attempted. The outcome of patients treated with combined chemotherapy and radiation in locally advanced disease is quite poor. In this context, inherent or acquired resistance mechanism operates to further reduce therapeutic efficacy. Better understanding of the nature of these resistance mechanisms appears to be of central importance towards the goal of developing more effective and less toxic therapy for lung cancer.

Doxorubicin (DOX) (Fig. 1A) is a highly active agent in the chemotherapeutic treatment of a number of human malignancies including breast cancer, bladder cancer, multiple myeloma, lymphoma and small cell lung cancer (SCLC) $(3,4)$. In contrast to its high activity against SCLC, it is relatively ineffective against non-small cell lung cancer (NSCLC), which represents about 4/5 of all lung cancers. Even in SCLC, acquired resistance to DOX is common, accounting for the frequent failure to cure SCLC with DOXbased chemotherapy regimens. The resistance mechanisms responsible for inherent DOX-resistance of NSCLC and acquired DOX-resistance in SCLC have been the subject of numerous investigations (5). The inherently lower cellular accumulation of DOX in NSCLC as compared with SCLC has been frequently attributed as a major cause of DOXresistance in NSCLC. Increased protein kinase C (PKC) particularly PKC $\alpha$ activity is closely linked with DOXaccumulation defective resistance. The mechanism through which PKC $\alpha$ can influence cellular DOX-accumulation remains unclear since regulation of ATP Binding Cassette (ABC) transporter activity by PKC $\alpha$ has not been established. $\mathrm{PKC} \alpha$ has been shown to phosphorylate and stimulate the 
activity of Ral-interacting protein (RLIP76) (6-8), a novel non-ABC-transporter of DOX (9). In recent years, we have shown RLIP76, a non-ABC transporter to be involved in the inherent DOX resistance in NSCLC and acquired resistance in SCLC.

In the present review, we will discuss the studies which demonstrate that RLIP76 is a major DOX transporter and is a primary determinant of DOX resistance in lung cancer cells. We will also discuss the structural motifs of RLIP76 and some of the interacting proteins which affect its transport function and conclude with a discussion of the implication of our novel finding in understanding the mechanisms of drug resistance in lung cancer cells for the development of pharmacological methods for the treatment of lung cancer.

\section{RLIP76: a non-ABC transporter}

RLIP76 is a stress-responsive, multi-functional membrane protein found ubiquitously in species as distant as Drosophila to humans (8-14). It is encoded in humans on chromosome 18 p11.3 by a gene with 11 exons and 9 introns (15). RLIP76 is a $76 \mathrm{kDa}$ protein product of this gene, but splice-variants include a $67 \mathrm{kDa}$ peptide and longer 80 or $102 \mathrm{kDa}$ peptide, (later identified as cytocentrin) $(16,17)$. RLIP76 is frequently overexpressed in malignant cells, and plays a prominent antiapoptotic role selectively in cancer cells through its ability to control cellular concentration of pro-apoptotic oxidized lipid by-products (18).

RLIP76 does not meet the criteria for classical ABC transporters as it does not have significant sequence homology with $\mathrm{ABC}$ transporters and differs from the $\mathrm{ABC}$ transporters in several other important aspects, including (i) lack of a classical Walker domain, (ii) lack of any close homologs in humans, (iii) integral membrane association without clearly defined transmembrane domains and (iv) its role as a direct link to Ras/Ral/Rho and EGF-R signaling through its multifunctional nature, including GAP activity, regulation of exocytosis as well as clathrin-coated pit-mediated receptor endocytosis $(8,13)$.

Although the primary amino acid sequence of RLIP76 is known $(8,10)$, detailed three-dimensional structure for the molecule remains to be elucidated. This knowledge will be important in understanding the transport functions as well as other activities of RLIP76. The protein lacks the well-defined trans-membrane helices of $\mathrm{ABC}$ transporters and there are no other RLIP76 family members with solved structure. Nevertheless, we can estimate domain behavior from sequence analysis and mutational studies. There are two ATP binding sites, one at the $\mathrm{NH}_{2}$ terminus $\left({ }^{69} \mathrm{GKKKGK}^{74}\right)$ and the other at the $\mathrm{COOH}$ terminus $\left({ }^{418} \mathrm{GGIKDLSK}^{425}\right)$ (19). Both these sites are needed for substrate transport. Mutations in these sequences abrogate its ATP-binding, ATPase activity and transport function $(19,20)$. Primary structure of RLIP76 can be divided into four regions. $N$ terminal domain (aa 1-209), is rich in antennapedia homeodomain homologous peptides. These peptides are known to be capable of transporting wide range of substrates across membranes. The $\mathrm{N}$-terminal domain also provides the membrane anchor. It has $\mathrm{N}$ myristolation sites at amino acid 21-26, 40-45, and 191-196. There are vector peptide-related sequences between amino acid 154 and 219 including the penetratin-like sequence ${ }^{154}{ }^{5 T A A D V V K Q W K E K K K K K ~}{ }^{170}$, a TAT-like sequence from amino acid 170-185, and a hydrophobic stretch from amino acid 203-219. Both membrane association and transport requires this domain $(8,20,21)$. Two central domains include, one carrying a Rac/cdc42 GAP (through aa 210-357) activity, while the other binds to activated Ral (aa 391-499). A Cterminal coiled-coil domain (aa 500-655) has subsequently been shown to interact with Reps1/POB1, CDK1 and Hsf-1 (Fig. 1B). These structural features link RLIP76 to a confounding array of physiological functions including clathrin-coated-pit-mediated receptor-ligand endocytosis of epidermal growth factor receptor (EGFR), insulin receptor (IR) and transforming growth factor $\beta$ (TGF- $\beta$ ) as well as mitosis signaling $(8,16,21-27)$. In a series of experiments over the last decade, an important role for RLIP76 in malignant cell survival and drug resistance and normal tissue response to oxidative stress has been confirmed.

RLIP76 was cloned originally as a Ral-binding GTPaseactivating protein (GAP), and predicted to be a Ral-effector, through a yeast two-hybrid screen (10). We cloned RLIP76 independently, in the search for glutathione-electrophile conjugates (GS-Es) and chemotherapy drug transporters, from a human bone-marrow cDNA library using polyclonal antibodies raised against purified human erythrocyte putative membrane transporter, DNPSG-ATPase (28-30). The identity of RLIP76 with DNPSG-ATPase was demonstrated by showing that both the human and recombinant protein could be purified by the same GS-E affinity chromatography method (9,28-30). Tissue-purified and recombinant RLIP76 have demonstrated constitutive ATPase activity that was stimulated by anionic (e.g., DNP-SG, GS-HNE,) as well as cationic (e.g., DOX) ligands $(9,31)$. In both cells and reconstituted proteoliposomes, native and recombinant RLIP76 can transport these allocrites across membranes (9). Transport activity of purified RLIP76 fraction was only minimally affected by limited proteolytic digestion, suggesting that intact RLIP76 was not necessary for transport and that peptides originating from RLIP76 could constitute a functional transporter $(9,19)$. This postulated hypothesis was confirmed by comparing the transport activity of intact RLIP76 with that of the N-terminal (N-RLIP76 ${ }^{1-367}$ ) and Cterminal (C-RLIP76 ${ }^{410-655}$ ) fragments of RLIP76 obtained from expressing the corresponding cDNA in E. coli and purifying these fragments using a His-tag (19). Remarkably, when both peptides purified separately were reconstituted together in artificial liposomes, transport activity was nearly equal to that of intact recombinant RLIP76 (19). These results confirmed the postulate that peptides originating from RLIP76 could reconstitute a functional transporter.

\section{Physiological significance of RLIP76}

One of the physiologic functions of RLIP76 is regulation of intracellular concentration of the electrophilic intermediates of oxidative lipid metabolism by mediating efflux of GS-E formed from oxidative degradation of arachidonic acid, including leukotrienes and the 4HNE-GSH conjugates. RLIP76 mediated transport of amphiphilic chemotherapeutic agents such as anthracyclines and vinca alkaloids as well as 
A<smiles>COc1cccc2c1C(=O)c1c(O)c3c(c(O)c1C2=O)C[C@@](O)(C(=O)CO)C[C@@H]3O[C@H]1C[C@H](N)[C@@H](O)[C@H](C)O1</smiles>

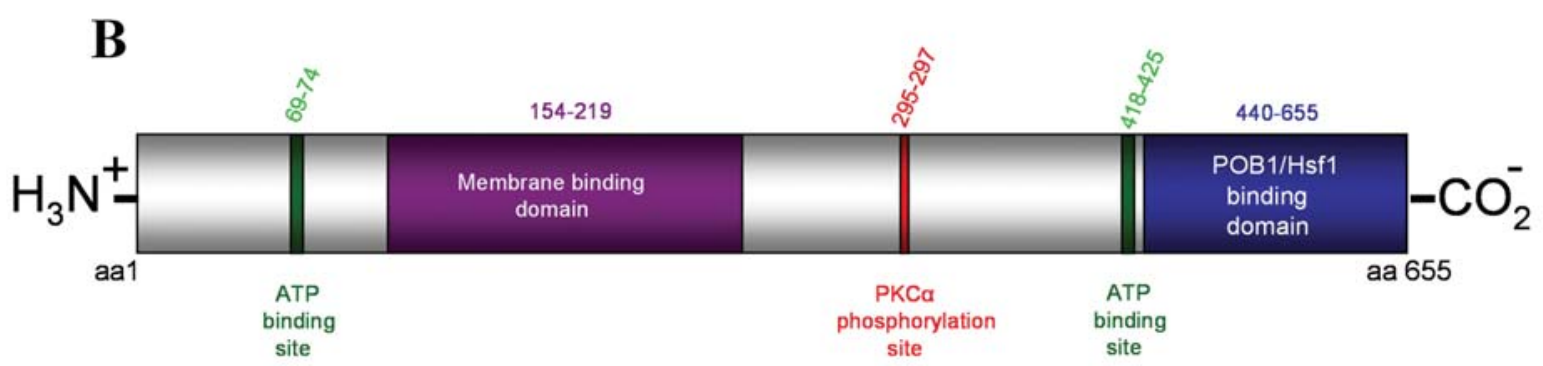

Figure 1. Structure of doxorubicin (panel A). Schematic representation of RLIP76 domains (panel B).

GS-E produced during oxidative metabolism places this multi-functional protein in a central role as a resistance mechanism for preventing apoptosis caused by chemotherapeutic agents. RLIP76 is also involved in variety of external/ internal stressors, including oxidative stress, heat shock and radiation $(25,32-34)$. Though RLIP76 is involved in many activities, in the present section we will discuss three major functions of RLIP76:

Protection against heat-shock and oxidative stress. Induction of RLIP76 was an early response to heat shock or oxidants, prior to the appearance of previously known heat shock proteins or antioxidant enzymes (32). When K562 cells were exposed to mild heat shock $\left(42^{\circ} \mathrm{C}, 30 \mathrm{~min}\right)$ or oxidative stress (50 $\mu \mathrm{M} \mathrm{H}_{2} \mathrm{O}_{2}, 20 \mathrm{~min}$ ) and allowed to recover for $2 \mathrm{~h}$, there was an enhanced level of $4 \mathrm{HNE}$, a several fold induction of hGST 5.8, which catalyzes the formation of GS-HNE, and induction of RLIP76, which mediates the transport of GSHNE from cells. Cells preconditioned with stress shock, transported GS-HNE at a 3-fold higher rate, and increased efflux of GS-HNE was observed. The increased efflux of GS-HNE was blocked by coating the cells with antibodies against RLIP76, confirming that GS-HNE is transported by RLIP76. The stress-preconditioned cells with induced hGST 5.8 and RLIP76 acquired resistance to $4 \mathrm{HNE}$ and $\mathrm{H}_{2} \mathrm{O}_{2}$ mediated apoptosis by suppressing sustained activation of JNK and caspase- 3 . The protective effect of stress preconditioning against $\mathrm{H}_{2} \mathrm{O}_{2}$ or $4 \mathrm{HNE}$ induced apoptosis was abrogated by coating the cells with anti-RLIP76 IgG, which inhibited the efflux of GS-HNE. These results showed that the cells acquired resistance to apoptosis by metabolizing and excluding $4 \mathrm{HNE}$ at a higher rate and suggested that the intracellular concentrations of GS-HNE and endogenous GS-E modulated stress-mediated signaling (32).
Transport mediated drug-resistance. RLIP76 serves an antiapoptotic function by preventing accumulation of endogenously derived GS-E. Endogenous GS-E, which forms as a result of oxidant or radiant stress, compete for efflux with a number of chemotherapy drugs including DOX, daunorubicin, colchicine, vincristine, vinblastine and vinorelbine, GS-E derived from alkylating agents or platinum coordinates which are known substrates for transport by RLIP76 $(8,9,28,31,35-37)$.

The role of RLIP76 in mediating DOX resistance was subsequently tested in K562 human leukemia cells by comparing the effect of transfection of empty vector vs. vector-containing full-length RLIP76 cDNA. RLIP76 was overexpressed 4-fold in Western blots as well as by RTPCR analysis. The DOX and DNP-SG transport activity of RLIP76-transfected cells increased $\sim 5$-fold and $\mathrm{IC}_{50}$ of DOX increased approximately 5-fold. As would be expected for a GS-E transporter, the $\mathrm{IC}_{50}$ for $4 \mathrm{HNE}$, an endogenous alkylating agent, also increased approximately 3-fold. The effect of increased RLIP76 expression was also evaluated by increasing RLIP76 in cells by delivering it using proteoliposomes containing RLIP76. H358 lung cancer cells showed avid uptake of RLIP76, with cellular RLIP76 protein increasing in a linear manner with respect to the amount of RLIP76 proteoliposomes used. Resistance to DOX increased in a linear fashion with respect to the amount of RLIP76 in proteoliposomes $(9,38)$.

RLIP76 protects against radiation toxicity. Since generation of reactive-oxygen species (ROS) is a common pathway for highenergy radiation as well as heat shock and oxidant exposure, we summarized that RLIP76 would also afford protection against radiation. We tested this hypothesis by determining the effect of increased RLIP76 on radiation-sensitivity. Because 
we had previously demonstrated that RLIP76 containing proteoliposomes were avidly taken up by cultured cells and would result in an increased cellular RLIP76 level as well as DOX resistance, we treated SCLC cells (H182 and H1618) in culture with liposome reconstituted in the presence of purified RLIP76 prior to the exposure to a single dose of radiation at 500 cGy. After radiation, cells were serially cultured by reinoculation into medium each day at a density of $5 \times 10^{5}$ cells $/ \mathrm{ml}$, and cell density was determined at $24 \mathrm{~h}$ intervals. Cells enriched with RLIP76 were significantly more resistant to radiation compared to control cells. These results suggested that accumulation of GS-E formed from reactive intermediates of lipid peroxidation contributes significantly to radiation toxicity and that increased efflux of these by-products rendered cells resistant to radiation $(8,22)$.

\section{Doxorubicin resistance in lung cancer}

DOX, an anthracycline antibiotic, is one of the most widely used anticancer drugs, effective in a wide variety of human cancers (39-41). Its efficacy, like that of other anticancer drugs, is hampered by innate and acquired drug resistance in cancer cells. Multiple mechanisms of DOX resistance have been elucidated. Although alterations in cellular antioxidant defenses (42), topoisomerases (43), and stress and apoptosis signaling pathway proteins (44) are found in many DOXresistant cells, drug-efflux mechanisms which create a DOXaccumulation defect appears to be the most common alteration $(44,45)$. The ABC-family of multi-specific transporters including P-glycoprotein (Pgp, ABCB1), multidrugresistance protein (MRP1, $\mathrm{ABCC} 1)$, and multiple other MRP-related transporters have been shown to mediate DOXtransport and resistance (46-48). Pgp was the first to be identified among these transporters (49), and differed from MRP and related transporters in that the later transporters use not only the weakly cationic anthracycline and vinca alkaloids as substrates, but can also transport GS-E of endogenous and exogenous alkylating agents (including a number of chemotherapy drugs). Though the ABC-transporters play a significant role in protecting many cancer cell types from DOX, in vitro and clinical studies indicate the involvement of other DOX-transporters as well $(49,50)$. Multiple potential mechanisms for DOX-efflux have been identified in lung cancer cells, primarily belonging to the ABC-transporter super-family, though rigorous evidence for DOX-transport activity in isolated systems is lacking for most. $\mathrm{P}$-glycoprotein (mdr-1 gene product, $\mathrm{Pgp}$ ) is the prototypical and best characterized membrane protein which has been shown to mediate the ATP-dependent transmembrane transport of DOX, as is a major cause of a DOX-accumulation defective phenotype in many types of human cancers, but its role in inherent resistance to DOX in lung cancer is likely limited because of its relatively low expression in lung cancer cells (51). Other ABC-super family transporters such as the MRP1 (ABCC1, multidrug-resistance associated protein) and BCRP (ABCG2) are more significant determinants of DOX transport in lung cancer cells $(51,52)$. However, our recent studies in panels of SCLC and NSCLC have shown that RLIP76 contributes approximately $2 / 3$ of total DOX-transport activity with the remainder being contributed to by $\mathrm{ABC}$-transporter (51).

\section{RLIP76 is a major DOX transporter}

Our laboratory showed for the first time, that RLIP76 can mediate ATP-dependent transmembrane movement of not only anionic GS-E (DNP-SG), but also weakly cationic chemotherapeutic drug (DOX) in several independent systems $(8,9)$. This was proved by experimental evidence such as i) transport studies on proteoliposomes (reconstituted with either asolectin or defined phospholipids) containing purified recombinant RLIP76; ii) acquired resistance to DOX and $4 \mathrm{HNE}$ in the cells transfected with cDNA encoding RLIP76; iii) acquired resistance to DOX in the cells upon direct incorporation of RLIP76 by fusion of the cells with proteoliposomes. These results confirmed and indicated that RLIP76, in addition to its role in signal transduction, can catalyze transport of GS-E and xenobiotics, and may contribute to the multidrug resistance phenomenon. In a variety of transport studies, we have proved that DOX is an excellent substrate for ATP-dependent efflux by RLIP76. Because drug-efflux mechanisms mediate drug accumulation defects as well as resistance to the cytotoxic effects of drugs, the function of RLIP76 as a drug transporter would predict that overexpression of this protein should render resistance to the drugs which are its allocrites. We initially addressed this hypothesis by comparing DOX sensitivity with the expression of RLIP76 as well as transport activity between wild-type and DOX exposure derived, resistant H69 SCLC cell lines. The 3- and 30-fold DOX-resistant variants tested had similar levels of MRP and undetectable levels of Pgp. Reconstituted RLIP76 catalyzes ATP-dependent uptake of ${ }^{14} \mathrm{C}$-DOX into vesicles. The activation energy for transport of the DOX was $96 \mathrm{~kJ} / \mathrm{mol}$, and the temperature optimum was at $37^{\circ} \mathrm{C}$ with inactivation at $45^{\circ} \mathrm{C}$. The calculated kinetic parameters for DOX transport in reconstituted RLIP76 are as follows: $\mathrm{K}_{\mathrm{m}}$ for ATP, $4.6 \mathrm{mM} ; \mathrm{K}_{\mathrm{m}}$ for DOX, $3.1 \mu \mathrm{M}$; $k$ cat for DOX (assuming half of the protein was reconstituted in transport-competent orientation), $1.9 \mathrm{~s}^{-1}$ (9).

To prove further, we generated RLIP76 ${ }^{+/+}$and RLIP76 ${ }^{-1-}$ mice colonies, based on genotyping (33). Replacement of RLIP76 in RLIP76-- mouse tissues were examined in animals given $200 \mu \mathrm{g} / 0.2 \mathrm{ml}$ purified RLIP76 liposomes, i.p. RLIP76 was clearly detected in mouse tissues at $24 \mathrm{~h}$ after an RLIP76-liposomes dose (33). Consistent with the observed function of RLIP76 as a transporter of DOX in cell culture studies (51), DOX transport in membrane vesicles was found to be decreased in RLIP76-- mice tissues. A greater than $80 \%$ loss of GS-E and DOX transport activity was seen in the

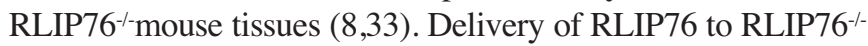
animals via liposomal delivery system completely reversed transport mechanisms $(33,53)$. Greater than $80 \%$ loss in liver and heart tissue of total DOX and GS-E transport activity in RLIP76 knockout mice strongly supports the hypothesis that RLIP76 can contribute substantially to total DOX transport capacity in cancer cells. Sequence analysis of RLIP76 indicates the presence of four potential PKC $\alpha$ phosphorylation sites (8). Since increased PKC $\alpha$ activity is correlated with increased DOX-resistance and decreased cellular DOXaccumulation, and because RLIP76 can be activated through phosphorylation by PKC $\alpha$ (7), mutational studies in RLIP76 at $\mathrm{PKC} \alpha$ phosphorylation sites proved that the regulation of 
RLIP76 by PKC $\alpha$ is a significant determinant of inherent and acquired DOX-resistance in lung cancer $(7,54)$.

\section{Impairment of transport of RLIP76 sensitizes lung cancer cells to doxorubicin}

Our studies proved that RLIP76 is a major transporter of DOX. If this assertion were true, inhibition of the transport function of RLIP76 after treatment with RLIP76 antibody should significantly enhance the cytotoxicity of DOX in these cells. Using highly specific anti-RLIP76 IgG at the concentration of $50 \mu \mathrm{g} / \mathrm{ml}$, we treated both NSCLC and SCLC for $16 \mathrm{~h}$ followed by addition of DOX in these cells. Our studies confirmed that anti-RLIP76 antibody markedly enhanced the cytotoxicity of DOX in NSCLC and SCLC. Further increase in IgG concentration showed saturation, which is consistent with a specific antigen and antibody interaction. The interaction of anti-RLIP76 IgG and DOX was markedly synergistic (CI $0.36 \pm 0.17$ ). The degree of synergy between anti-RLIP76 IgG and DOX was found to be significantly greater (CI near 0.001 ) than that between DOX and Herceptin (an anti-Her-2/neu humanized monoclonal antibody used in therapy of human breast cancer). Overall, the sensitizing effect of anti-RLIP76 IgG to DOX was somewhat more prominent for SCLC as compared to NSCLC. Mechanism on anti-RLIP76 IgG mediated cytotoxicity was explained by a model built on our previous studies in which a physiologic function (i.e. GS-E efflux) of RLIP76 exerts an anti-apoptotic effect, and DOX inhibits this physiologic function. Our studies showed that specific inhibition of the transport function of RLIP76 by RLIP76 IgG can trigger apoptosis and can increase DOX cytotoxicity synergistically (55).

\section{Role of PKC $\alpha$ and RLIP76 in transport-mediated DOX-resistance in lung cancer}

In order to answer the question whether PKC $\alpha$ or RLIP76 or both are the cause for differential drug-resistance observed in NSCLC and SCLC, we determined the expression of PKCa and RLIP76 in several NSCLC and SCLC cell lines. Western blot analysis using antibodies against $\mathrm{PKC} \alpha$, with equal amount of crude protein from either cell line indicated a higher expression of PKC $\alpha$ in all NSCLC cell lines as compared to SCLC cell lines. Depletion of PKC $\alpha$ by siRNA, in several NSCLC and SCLC cell lines, showed a decrease in the drug resistance $\left(\mathrm{IC}_{50}\right.$ for DOX) of NSCLC cell lines to the level observed with SCLC cell lines. Same phenomenon was observed in DOX-transport studies using proteoliposomes reconstituted with purified RLIP76 from PKC $\alpha$-depleted NSCLC and SCLC. These results directly indicate that PKC $\alpha$ plays a major role towards differential DOX-resistance of NSCLC and SCLC $(7,54,56)$.

The difference in RLIP76 activity between NSCLC and SCLC is not related to the amount of RLIP76 protein in these cells rather to the specific activity of RLIP76. Our studies showed that specific activity of highly purified RLIP76 from six SCLC cell lines were approximately half that observed for seven NSCLC. SCLC and NSCLC have inherently different sensitivity to DOX, SCLC being significantly more sensitive than NSCLC, our findings for the first time suggested that differences in RLIP76 between NSCLC and SCLC plays a significant role in inherent difference in DOX sensitivity in these two distinct types of lung malignancy $(17,51,55)$.

Further, we investigated the mechanisms how RLIP76 activity is higher in NSCLC as compared with SCLC by sitedirected mutagenesis studies of RLIP76 gene. Sequence analysis of RLIP76 indicated the presence of four $\left(\mathrm{S}^{118}, \mathrm{~T}^{297}\right.$, $\mathrm{S}^{353}$ and $\mathrm{S}^{509}$ ) potential PKC $\alpha$ phosphorylation sites $(7,54)$. In deletion mutant analyses of potential phosphorylation sites in RLIP76, we identified $\mathrm{T}^{297}$ and $\mathrm{S}^{509}$ as targets for phosphorylation by $\mathrm{PKC} \alpha$. Phosphorylation at $\mathrm{T}^{297}$ increased DOXtransport activity by 2 -fold for RLIP76 purified from recombinant source, or from three SCLC (H69, H1417 and H1618) and three NSCLC, one each derived from H226 (squamous), H358 (bronchio alveolar) and H1395 (adenocarcinoma). ATP-dependent transport of DOX for each of these mutants indicated that PKC $\alpha$ can activate RLIP76, and that the majority of this effect is abrogated by deletion of $\mathrm{T}^{297}$.

In our initial studies (17), we have found that both SCLC and NSCLC cell lines contain similar amounts of RLIP76 protein $(0.53 \pm 0.04 \%$ of total membrane protein, $n=12)$, but the specific activity of RLIP76 from NSCLC was $\sim 2$-fold greater than from SCLC, both in ATPase activity and transport activity. Post-translational modifications as well as differences in susceptibility to limited tryptic digestion were suggested to be the reason for this difference. Our studies showed the presence of a $44 \mathrm{kDa}$ peptide resulting from limited proteolytic digestion of RLIP76 from NSCLC, which was absent from SCLC. The N-terminal sequence of this $44 \mathrm{kDa}$ peptide revealed the sequence TTETE, beginning at $\mathrm{T}^{294}$. Pretreatment of the protein from SCLC with PKC $\alpha$ caused the appearance of this band. The $44 \mathrm{kDa}$ band was not seen upon PKC $\alpha$ pre-treatment of rec-RLIP76 del ${ }^{297-299}$. These findings indicate that in NSCLC, RLIP76 exists predominantly as a phosphorylated form at $\mathrm{T}^{297}$, and its characteristic susceptibility to serine protease at $\mathrm{T}^{294}$ confers a significantly greater DOXtransport activity (7).

\section{RLIP76 is essential for PKC $\alpha$ mediated DOX-resistance}

The role of RLIP76 in PKC $\alpha$-mediated resistance signaling was analyzed by determination of $\mathrm{IC}_{50}$ for DOX in RLIP76 ${ }^{+/+}$ and RLIP76 ${ }^{-1-}$ mouse embryonic fibroblasts (MEFs) in the presence and absence of PKC $\alpha$. Although RLIP76-- MEFs were significantly more sensitive to DOX as compared to RLIP76 $^{+/+}$MEFs ( IC $_{50} 25$ vs. $125 \mathrm{nM}$ ), depletion of PKC $\alpha$ only affected the DOX-sensitivity of RLIP76 ${ }^{+/+}$MEFs by 2.2-fold. These results demonstrate that the effect of PKC $\alpha$ mediated drug resistance requires the presence of functional RLIP76; that is, the primary function of PKC $\alpha$ with respect to DOX-resistance in NSCLC is to keep RLIP76 in its active phosphorylated state. The complete reversion of the resistance phenotype from NSCLC to that of SCLC upon depletion of PKC $\alpha$ and the complete inability of PKC $\alpha$ to affect resistance in RLIP76 ${ }^{-/-}$MEFs strongly supports this conclusion.

To find a solution to the important question whether RLIP76 is upstream or downstream of PKC $\alpha$-mediated pathway, we depleted the PKC $\alpha$ in MEFs. Depletion of PKC $\alpha$ 
causes 30\% decrease in rate of proliferation of MEFs. This decrease is absent in RLIP76 gene knockout (RLIP76 ${ }^{-1-}$ ) MEFs. PKC $\alpha$ stimulate DOX-resistance by increased efflux, in RLIP76 ${ }^{+/+}$MEFs while RLIP76 ${ }^{-/-}$MEFs lack this phenotype completely. These results lead to the definitive conclusion that RLIP76 is a downstream regulator of PKC $\alpha$ and is required for $\mathrm{PKC} \alpha$-mediated signal proliferation $(54,56)$.

\section{RLIP76 inhibition by POB1 and Hsf-1 induces DOX sensitivity}

POB1 (partner of RLIP76), also known as REPS2, was discovered in screening for the RLIP76-binding proteins using the yeast two-hybrid system along with REPS 1 $(24,25,57)$. Hsf-1 (heat shock factor-1) is a transcription factor that is known to regulate cellular heat shock response through its binding with the multi-specific transporter protein, RLIP76 $(23,25)$. In the unstressed state, Hsf-1 is sequestered in a complex with tubulin, HSP90 and RLIP76 (23). Stress or constitutively active Ral-GTP binding to RLIP76 triggers the release of Hsf-1 and its migration to the nucleus, where its transcription factor activity is important for the expression of heat shock proteins (25).

Since inhibition or depletion of RLIP76 has been shown previously to selectively target lung cancer cells for apoptosis and sensitize them to DOX toxicity (58), we reasoned that augmenting cellular POB1 or Hsf-1 should inhibit efflux of DOX from NSCLC, resulting in increased accumulation and thus increased cytotoxicity of DOX. POB1 caused a saturable inhibition of ${ }^{14} \mathrm{C}$-DOX transport with maximal inhibition of about $50 \%$. The addition of Hsf-1 also caused saturable inhibition of transport, with a maximum of $40 \%$. In the presence of both proteins, $90 \%$ inhibition of ${ }^{14} \mathrm{C}$-DOX transport was observed. The additive nature of transport inhibition is consistent with distinct but perhaps overlapping binding sites for POB1 ( $\left.\mathrm{aa}^{499-655}\right)$ and Hsf-1 $\left(\mathrm{aa}^{440-655}\right)$, both being present in the $\mathrm{C}$-terminal region (25). These striking findings predict that simultaneous augmentation of POB 1 and Hsf-1 would very effectively inhibit RLIP76 transport activity.

\section{Conclusions}

Studies summarized here demonstrate that RLIP76 is a primary determinant of DOX-resistance, and that PKC $\alpha$ mediated accumulation defect and DOX-resistance in NSCLC is primarily due to differential phosphorylation of RLIP76 in SCLC and NSCLC. Depletion of RLIP76 in these cells not only triggers apoptosis but also synergistically increases DOX efficacy. These studies are of great importance in deciding the treatment for lung cancers as well as future drug development. RLIP76 phosphorylation or RLIP76 expression can be used as a marker which could help in selecting patients and predicting clinical response. Humanization of a monoclonal antibody or antisense seems the most direct route. Another application of the RLIP76 technology is to increase its levels in cells by delivery of RLIP76-loaded proteoliposomes which may reduce side effects of cancer therapy. Hsf-1 and POB1 augmentation in lung cancer cells inhibit RLIP76 transport activity; their liposomally encapsulated form could also be used clinically as a therapeutic agent. Development of these treatment modalities will require adequate production of RLIP76 and formulation for both regional and systemic delivery. As discussed above, RLIP76 is a major drug transporter in conferring drug resistance in lung cancer and inherent resistance in NSCLC, future drugs with poor transport through RLIP76 will be more efficient in skirmishing against lung cancer.

\section{Acknowledgements}

This study was supported in part by NIH Grants CA 77495, CA 104661 and ES 012171, Cancer Research Foundation of North Texas, Institute for Cancer Research and the Joe and Jessie Crump Fund for Medical Education.

\section{References}

1. Arriagada R, Le Péchoux C and Pignon JP: Resected non-small cell lung cancer: need for adjuvant lymph node treatment? From hope to reality. Lung Cancer 42: S57-S64, 2003.

2. Hoffman PC, Mauer AM and Vokes EE: Lung cancer. Lancet 355: 479-485, 2000.

3. Dalpiaz O, al Rabi N, Galfano A, Martignoni G, Ficarra V and Artibani W: Small cell carcinoma of the bladder: a case report and a literature review. Arch Esp Urol 56: 197-202, 2003

4. Filipits M, Pohl G, Stranzl T, et al: Low p27Kip1 expression is an independent adverse prognostic factor in patients with multiple myeloma. Clin Cancer Res 9: 820-826, 2003.

5. Volm M, Koomagi R and Rittgen W: Cellular predictive factors of drug resistance in non-small cell lung carcinomas. Methods Mol Med 75: 39-51, 2003.

6. Pikula S, Hayden JB, Awasthi S, Awasthi YC and Zimniak P: Organic anion-transporting ATPase of rat liver. I. Purification, photoaffinity labeling, and regulation by phosphorylation. J Biol Chem 269: 27566-27573, 1994.

7. Singhal SS, Yadav S, Singhal J, Drake K, Awasthi YC and Awasthi S: The role of PKC $\alpha$ and RLIP76 in transport-mediated doxorubicin-resistance in lung cancer. FEBS Lett 579: 4635-4641, 2005.

8. Awasthi S, Singhal SS, Sharma R, Zimniak P and Awasthi YC: Transport of glutathione-conjugates and chemotherapeutic drugs by RLIP76: a novel link between G-protein and tyrosine kinase signaling and drug resistance. Int J Cancer 106: 635-646, 2003.

9. Awasthi S, Cheng J, Singhal SS, et al: Novel function of human RLIP76: ATP-dependent transport of glutathione-conjugates and doxorubicin. Biochemistry 39: 9327-9334, 2000.

10. Jullien-Flores V, Dorseuil O, Romero F, et al: Bridging RalGTPase to Rho-pathways. RLIP76, a Ral-effector with CDC42/ Rac GTPase-activating protein activity. J Biol Chem 270: 22473-22477, 1995.

11. Cantor SB, Urano T and Feig LA: Identification and characterization of Ral-binding-protein1, a potential downstream target of Ral GTPases. Mol Cell Biol 15: 4578-4584, 1995.

12. Park SH and Weinberg RA: A putative effector of Ral has homology to Rho/Rac GTPase activating proteins. Oncogene 11: 2349-2355, 1995.

13. Jullien-Flores V, Mahe Y, Mirey G, et al: RLIP76, an effector of the GTPase Ral, interacts with the AP2 complex: involvement of the Ral pathway in receptor-endocytosis. J Cell Sci 113: 2837-2844, 2003.

14. Mirey G, Balakireva M, L'Hoste S, Rossé C, Voegeling S and Camonis J: A Ral guanine exchange factor-Ral pathway is conserved in Drosophila melanogaster and sheds new light on the connectivity of the Ral, Ras and Rap-pathways. Mol Cell Biol 23: 1112-1124, 2003.

15. www.ensembl.org human/exon report.

16. Quaroni A and Paul EC: Cytocentrin is a Ral-binding protein involved in the assembly and function of the mitotic-apparatus. J Cell Sci 112: 707-718, 1999.

17. Singhal SS, Singhal J, Sharma R, et al: Role of RLIP76 in lung cancer doxorubicin-resistance: I The ATPase activity of RLIP76 correlates with doxorubicin and 4HNE-resistance in lung cancer cells. Int J Oncol 22: 365-375, 2003. 
18. Singhal SS, Awasthi YC and Awasthi S: Regression of melanoma in a murine model by RLIP76 depletion. Cancer Res 66: 2354-2360, 2006.

19. Awasthi S, Cheng JZ, Singhal SS, et al: Functional reassembly of ATP-dependent xenobiotic transport by the N- and Cterminal domains of RLIP76 and identification of ATP binding sequences. Biochemistry 40: 4159-4168, 2001.

20. Yadav S, Singhal SS, Singhal J, et al: Identification of membraneanchoring domains of RLIP76 using deletion mutant analysis. Biochemistry 43: 16243-16253, 2004.

21. Yadav S, Zajac E, Singhal SS and Awasthi S: Linking stresssignaling, glutathione metabolism, signaling pathways and xenobiotic transporters. Cancer Metastasis Rev 26: 59-69, 2007.

22. Awasthi S, Sharma R, Singhal SS, Zimniak P and Awasthi YC RLIP76, a novel transporter catalyzing ATP-dependent efflux of xenobiotics. Drug Metab Dispos 30: 1300-1310, 2002.

23. Hu Y and Mivechi NF: HSF-1 interacts with Ral-bindingprotein 1 in a stress-responsive, multi-protein complex with HSP90 in vivo. J Biol Chem 278: 17299-17306, 2003.

24. Oosterhoff JK, Penninkhof F, Brinkmann AO, Grootegoed JA and Blok LG: POB1 is down-regulated during human prostate cancer progression and inhibits growth factor signaling in prostate cancer cells. Oncogene 22: 2920-2925, 2003.

25. Singhal SS, Yadav S, Drake K, Singhal J and Awasthi S: Hsf-1 and POB1 induce drug-sensitivity and apoptosis by inhibiting Ralbp1. J Biol Chem 283: 19714-19729, 2008.

26. Rosse C, L'Hoste S, Offner N, Picard A and Camonis JH: RLIP76, an effector of the Ral-GTPases, is a platform for Cdk1 to phosphorylate epsin during the switch off of endocytosis in mitosis. J Biol Chem 278: 30597-30604, 2003.

27. Ikeda M, Ishida O, Hinoi T, Kishida S and Kikuchi AJ: Identification and characterization of a novel protein interacting with Ral-binding protein 1, a putative effector protein of Ral. J Biol Chem 273: 814-821,1998

28. Awasthi S, Singhal SS, Srivastava SK, et al: Adenosinetriphosphate-dependent transport of doxorubicin, daunomycin, and vinblastine in human tissues by a mechanism distinct from the P-glycoprotein. J Clin Invest 93: 958-965, 1994.

29. Awasthi S, Singhal SS, Srivastava SK, et al: ATP dependent human erythrocyte glutathione-conjugate-transporter. I Purification, photoaffinity-labeling, and kinetic characteristics of ATPase-activity. Biochemistry 37: 5231-5238, 1998

30. Awasthi S, Singhal SS, Pikula S, et al: ATP-dependent human erythrocyte glutathione-conjugate-transporter. II. Functional reconstitution of transport-activity. Biochemistry 37: 5239-5248, 1998.

31. Sharma R, Singhal SS, Cheng J, et al: RLIP76 is the major ATP-dependent transporter of glutathione-conjugates and doxorubicin in human erythrocytes. Arch Biochem Biophys 391: 171-179, 2001

32. Cheng J, Sharma R, Yang Y, et al: Accelerated metabolism and exclusion of 4-hydroxy-nonenal through induction of RLIP76 and hGST5.8 is an early adaptive response of cells to heat and oxidative-stress. J Biol Chem 276: 41213-41223, 2001.

33. Awasthi S, Singhal SS, Yadav S, et al: RLIP76 is a major determinant of radiation-sensitivity. Cancer Res 65: 6022-6028, 2005

34. Singhal J, Singhal SS, Yadav S, et al: RLIP76 in defense of radiation poisoning. Int J Rad Oncol Biol Phys 72: 553-561, 2008

35. Sharma R, Singhal SS, Wickramarachchi D, Awasthi YC and Awasthi S: RLIP76 mediated transport of leukotrienes C4 (LTC4) in cancer cells: implications in drug resistance. Int J Cancer 112: 934-942, 2004

36. Stuckler D, Singhal J, Singhal SS, Yadav S, Awasthi YC and Awasthi S: RLIP76 transports vinorelbine and mediates drugresistance in non-small cell lung cancer. Cancer Res 65: 991-998, 2005

37. Awasthi S, Singhal SS, Pandya U, Gopal S, Zimniak P, Singh SV and Awasthi YC: ATP-dependent colchicine transport by human erythrocyte glutathione-conjugate transporter. Toxicol Appl Pharmacol 155: 215-226, 1999.

38. Singhal SS, Sehrawat A, Metha A, Sahu M and Awasthi S: Functional reconstitution of RLIP76 catalyzing ATP-dependent transport of glutathione-conjugate. Int J Oncol 34: 191-199, 2009
39. Goldin A, Venditti JM and Geran R: The effectiveness of the anthracycline analog 4'-epidoxorubicin in the treatment of experimental tumors: a review. Invest New Drugs 3: 3-21, 1985.

40. Booser DJ and Hortobagyi GN: Anthracycline antibiotics in cancer therapy. Focus on drug resistance. Drug 47: 223-258, 1994.

41. Singal PK, Siveski-Iliskovic N, Hill M, Thomas TP and Li T: Combination therapy with probucol prevents adriamycininduced cardiomyopathy. J Mol Cell Cardiol 27: 1055-1063, 1995.

42. Yeh GC, Daschner PJ, Lopaczynska J, MacDonald CJ and Ciolino HP: Modulation of glucose-6-phosphate dehydrogenase activity and expression is associated with aryl hydrocarbon resistance in vitro. J Biol Chem 276: 34708-34713, 2001.

43. Zwelling LA, Slovak ML, Doroshow JH, et al: HT1080/DR4: a P-glycoprotein-negative human fibrosarcoma cell line exhibiting resistance to topoisomerase II-reactive drugs despite the presence of a drug-sensitive topoisomerase II. J Natl Cancer Inst 82: $1553-1561,1990$

44. Song SH, Lee KH, Kang MS and Lee YJ: Role of paxillin in metabolic oxidative stress-induced cytoskeletal reorganization: involvement of SAPK signals transduction pathway and PTPPEST gene expression. Free Radic Biol Med 29: 61-70, 2000.

45. Kok JW, Veldman RJ, Klappe K, Koning H, Filipeanu CM and Muller M: Differential expression of sphingolipids in MRP1 over-expressing HT29 cells. Int J Cancer 87: 172-178, 2000.

46. Borst P, Evers R, Kool M and Wijnholds J: The multidrug resistance protein family. Biochim Biophys Acta 1461: 347-357, 1999.

47. Cole SP, Bhardwaj G, Gerlach JH, et al: Over-expression of a transporter gene in a multidrug-resistant human lung cancer cell line. Science 258: 1650-1654, 1992

48. Gottesman MM and Pastan I: Biochemistry of multidrug resistance mediated by the multidrug transporter. Annu Rev Biochem 62: 385-427, 1993.

49. Lai SL, Goldstein LJ, Gottesman MM, et al: MDR1 gene expression in lung cancer. J Natl Cancer Inst 81: 1144-1150, 1989.

50. Hendrikse NH: Monitoring interactions at ATP-dependent drug efflux pumps. Curr Pharm Des 6: 1653-1668, 2000.

51. Awasthi S, Singhal SS, Singhal J, Cheng J, Zimniak P and Awasthi YC: Role of RLIP76 in lung cancer doxorubicin resistance II. Doxorubicin transport in lung cancer by RLIP76. Int J Oncol 22: 713-720, 2003

52. Singhal SS, Singhal J, Nair MP, Lacko AG, AwasthiYC and Awasthi S: Doxorubicin transport by RALBP1 and ABCG2 in lung and breast cancer. Int J Oncol 30: 717-725, 2007.

53. Singhal SS, Yadav S, Singhal J, Sahu M, Sehrawat A and Awasthi S: Diminished drug transport and augmented radiation sensitivity caused by loss of RLIP76. FEBS Lett 582: 3408-3414, 2008.

54. Singhal SS, Wickramarachchi D, Singhal J, Yadav S, Awasthi YC and Awasthi S: Determinants of differential doxorubicin sensitivity between SCLC and NSCLC. FEBS Lett 580: 2258-2264, 2006.

55. Awasthi S, Singhal SS, Singhal J, Yang Y, Zimniak P and Awasthi YC: Role of RLIP76 in lung cancer doxorubicin resistance III. Anti-RLIP76 antibodies trigger apoptosis in lung cancer cells and synergistically increase doxorubicin cytotoxicity. Int J Oncol 22: 721-732, 2003.

56. Singhal SS, Yadav S, Singhal J, Awasthi YC and Awasthi S: Mitogenic and drug-resistance mediating effects of $\mathrm{PKC} \alpha$ require RLIP76. Biochem Biophys Res Commun 348: 722-727, 2006.

57. Yadav S, Zajac E, Singhal SS, et al: POB1 over-expression inhibits RLIP76-mediated transport of glutathione-conjugates, drugs and promotes apoptosis. Biochem Biophys Res Commun 328: 1003-1009, 2005

58. Singhal SS, Yadav S, Singhal J, Zajac E, Awasthi YC and Awasthi S: Depletion of RLIP76 sensitizes lung cancer cells to doxorubicin. Biochem Pharmacol 70: 481-488, 2005. 